\title{
An Innovative Passive Residual Heat Removal System of an Open-Pool Type Research Reactor with Pump Flywheel and Gravity Core Cooling Tank
}

\author{
Kwon-Yeong Lee ${ }^{1}$ and Hyun-Gi Yoon ${ }^{2}$ \\ ${ }^{1}$ Handong Global University, 558 Handong-Ro, Heunghae-eup, Buk-gu, Pohang, Gyeongbuk 791-708, Republic of Korea \\ ${ }^{2}$ Korea Atomic Energy Research Institute, Daedeok-daero 989-111, Yuseong-gu, Daejeon 305-353, Republic of Korea \\ Correspondence should be addressed to Kwon-Yeong Lee; kylee@handong.edu
}

Received 6 July 2015; Accepted 5 August 2015

Academic Editor: Eugenijus Ušpuras

Copyright ( $\odot 2015$ K.-Y. Lee and H.-G. Yoon. This is an open access article distributed under the Creative Commons Attribution License, which permits unrestricted use, distribution, and reproduction in any medium, provided the original work is properly cited.

\begin{abstract}
In an open-pool type research reactor, the primary cooling system can be designed to have a downward flow inside the core during normal operation because of the plate type fuel geometry. There is a flow inversion inside the core from the downward flow by the inertia force of the primary coolant to the upward flow by the natural circulation when the pump is turned off. To delay the flow inversion time, an innovative passive system with pump flywheel and GCCT is developed to remove the residual heat. Before the primary cooling pump starts up, the water level of the GCCT is the same as that of the reactor pool. During the primary cooling pump operation, the water in the GCCT is moved into the reactor pool because of the pump suction head. After the pump stops, the potential head generates a downward flow inside the core by moving the water from the reactor pool to the GCCT and removes the residual heat. When the water levels of the two pools are the same again, the core flow has an inversion of the flow direction, and natural circulation is developed through the flap valves.
\end{abstract}

\section{Introduction}

Research reactors are generally divided into a tank-in-pool type and an open-pool type [1]. An open-pool type research reactor has a benefit from the viewpoint of reactor usage such as isotope production because the top of the core is opened. In an open-pool type research reactor, the decay heat of the core is transferred into the reactor pool by a natural circulation through the flap valves after the Primary Cooling Pump (PCP) is turned off. That is, the flap valves installed on the pipe of the Primary Cooling System (PCS) inside the reactor pool are designed to be passively opened, when the flow rate of the PCS is reduced below the design limit. If the core flow is in an upward direction during PCP operation, the transition from a forced convection to a natural convection will be natural, and concerns regarding the core damage will be very small. However, there is a recent trend to use a plate type fuel, and a core downward flow is considered because it is benefit to install a plate type fuel in the core. In this case, a flow inversion from a downward to upward flow in the core is generated after the PCP is turned off. If this phenomenon is introduced within a high heat flux region of residual heat, the fuel fails instantly due to a zero flow. Therefore, the core downward flow should be sufficiently maintained until the residual heat is in a low heat flux region.

In a small power research reactor, inertia generated by a flywheel of the PCP can maintain a downward flow shortly and resolve the problem of a flow inversion. However, a high power research reactor of more than $10 \mathrm{MW}$ should have an additional method to generate a longer downward flow until a low heat flux is reached. Usually, other research reactors have selected an active residual heat removal system as a safety class. In this case, it is difficult to design and operate an active system pump because its operating point varies according to the operation conditions of the PCP. In addition, the system should have two separate trains considering the redundancy and diversity and safety class UPS (Uninterruptible Power Supply) or EDG (Emergency Diesel Generator) considering a loss of electric power accident. 
In addition, the consumption of radioactive isotopes has recently increased for cancer diagnosis. However, isotope production research reactors around in the world are deteriorating and have low efficiency. Therefore, there are several plans to construct new research reactors for isotope production including the KJRR (KIJANG Research Reactor) of South Korea. A workplace to replace fuels and isotope targets is at the top of the reactor pool, and replacement works are difficult and take several days because of the heat waves by natural convection in the decay heat removal process. Thus, it is necessary to maintain a downward flow in the core with a low flow rate for the decay heat and heat wave removal.

\section{System Description}

2.1. Pool-Type Reactor Subgroups. DOE category III (pooltype) reactors are divided into two broad subgroups based on the maximum steady-state thermal power of the reactor [2]. The power ranges that define the subgroups are as follows:
(A) Power $\leq 1 \mathrm{MW}$,
(B) $1 \mathrm{MW}<$ Power $\leq 10 \mathrm{MW}$.

The first subgroup does not require active systems for reactor heat removal. Natural circulation is sufficient to cool the core. The second subgroup requires forced circulation to remove high reactor power ( $>1 \mathrm{MW})$. Additionally, a third subgroup operating at greater than $10 \mathrm{MW}$ thermal power is considered.

2.2. Pump Flywheel. A pump coastdown means a pump operation with the angular momentum of the shaft, impeller, and flywheel if a loss of off-site power occurs. At this time, the flow rate decreases slowly by the effect of the moment of inertia, and therefore, core cooling is continued smoothly.

Here, a modified hydraulic efficiency model for the PCP coastdown of the HANARO (High Flux Advanced Neutron Application ReactOr) was developed and evaluated with the field data [3]. The HANARO is a multipurpose research reactor in South Korea with the much expanded capability of neutron beam research, nuclear fuel and material irradiation, radioisotope production, neutron transmutation doping, neutron radiography, neutron activation analysis, and other basic researches. It has an open tank-in-pool for easy access and light water coolant. The moderator uses a combination of light water and heavy water, and the design thermal power is $30 \mathrm{MW}$. The type of the PCP of HANARO is a typical centrifugal pump with a specific speed of $315[\mathrm{rpm}$, $\left.\mathrm{m}^{3} / \mathrm{min}, \mathrm{m}\right]$ and specific diameter of $0.31\left[\mathrm{~m}, \mathrm{~m}^{3} / \mathrm{min}, \mathrm{m}\right]$. In this pump, a flywheel is connected with the rotating shaft and located at the back of the electric motor and its moment of inertia, $I$, is $156.9 \mathrm{~kg} \cdot \mathrm{m}^{2}$. The moment of inertia of the shaft and impeller was not considered in this simulation because its value was relatively small compared to that of the flywheel.

When a loss of off-site power occurs, the PCP starts to operate with the power of the flywheel. At this time, the flywheel has the angular momentum directly proportional to its moments of inertia and angular velocity of the pump impeller. The flywheel supplies the break horse power to the pump through the connected shaft. The initial supplied power

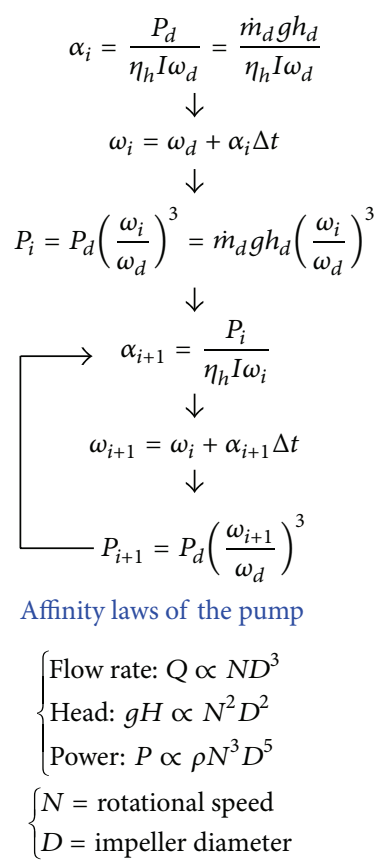

FIGURE 1: Calculation diagram of the coastdown.

was determined from the normal operation conditions of the pump. The mass flow rate, total head rise, angular velocity, and hydraulic efficiency of the normal operation are used as initial conditions to calculate the coastdown. Figure 1 shows the calculation diagram of the pump coastdown. Subscripts " $d$ " and " $i$ " in Figure 1 indicate normal operation conditions and the iteration number of calculations, respectively. This calculation flow chart explains the energy transform from the flywheel to the pump. It is assumed that the affinity laws of the pump are satisfied in the overall pump coastdown range and that the impeller diameter is a constant [4]. However, the hydraulic efficiency of the pump worsens when the impeller rotating speed is gradually decreased.

Generally, the hydraulic efficiency of the pump is related to the Reynolds number and impeller diameter as Pfleiderer's formula describes in [5]

$$
\begin{aligned}
\eta_{h}=1-\left(1-\eta_{d}\right)\left(\frac{\omega_{d}}{\omega_{i}}\right)^{0.1}\left(\frac{D_{d}}{D_{i}}\right)^{0.25}, & \\
\frac{1}{15} & <\frac{\omega_{i}}{\omega_{d}}<15 .
\end{aligned}
$$

The dotted line in Figure 2 shows the pump coastdown using this hydraulic efficiency equation based on the calculation diagram in Figure 1. The coastdown is not stopped after 100 seconds. However, the PCP coastdown of HANARO is continued until about 70-80 seconds from the field data. Thus, the hydraulic efficiency model for the row rotating speed compared to the normal operation is necessary to predict the coastdown accurately.

The decreases of the hydraulic efficiency of the pump during the coastdown are mainly related to disk friction loss of the pump and the increase of the system resistance curve 


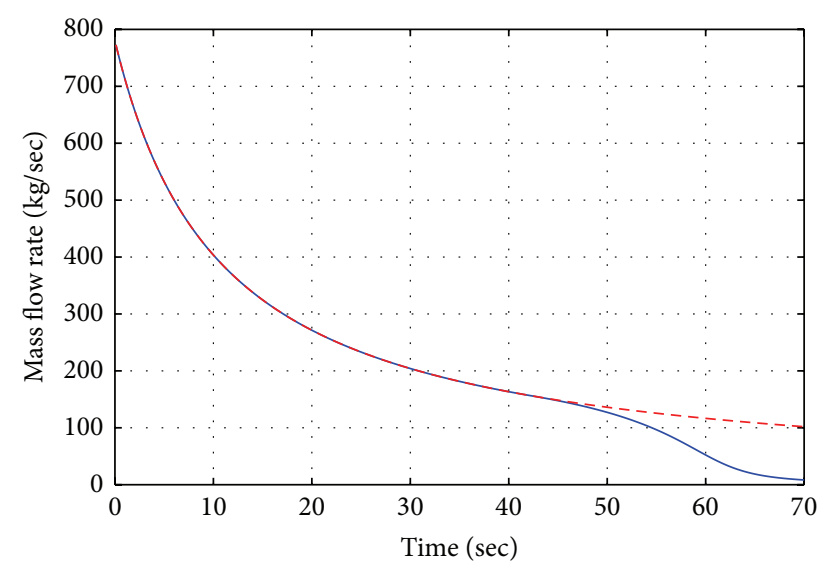

- Real case

- - - Ideal case

Figure 2: Coastdown of the pump with time (HANARO).

while the rotating speed is deceased [5-8]. The proportion of the disk friction loss to the pump head rise increases rapidly when the impeller rotating speed decreases as shown in Figure 3. In addition, the slope of the system resistance curve is steeper in the low flow rate region because of the increase of the friction loss. It shifts the operating point of the pump from the rated region to the low flow rate region, as described in Figure 4. These two factors decrease the hydraulic efficiency of the pump. In this research, the modified hydraulic efficiency model is suggested through (2) and (3) based on Pfleiderer's model. Consider

$$
\begin{gathered}
\eta_{h}=1-\left(1-\eta_{d}\right)\left(\frac{\omega_{d}}{\omega_{i}}\right)^{0.1}\left(\frac{D_{d}}{D_{i}}\right)^{0.25} \\
\text { when } 0.2<\frac{\omega_{i}}{\omega_{d}}<1, \\
\eta_{h}=1-\left(1-\eta_{d}\right)\left(\frac{\omega_{d}}{\omega_{i}}\right)^{0.1}\left(\frac{D_{d}}{D_{i}}\right)^{0.25} \\
\cdot \exp \left(-25\left(0.2-\frac{\omega_{i}}{\omega_{d}}\right)\right) \quad \text { when } 0<\frac{\omega_{i}}{\omega_{d}}<0.2 .
\end{gathered}
$$

In (3), a model constant of -25 is selected based on the coastdown time of HANARO. The solid line in Figure 2 shows the pump coastdown using this modified hydraulic efficiency equation. The pump stopped at about 70 seconds.

\subsection{Gravity Core Cooling Tank. A Passive Residual Heat} Removal System (PRHRS) for an open-pool type research reactor was developed using a Gravity Core Cooling Tank (GCCT), as shown in Figure $5[9,10]$. The GCCT beside the reactor pool is a small tank opening into the air with atmospheric pressure. A Residual Heat Removal Pipe (RHRP) is connected to a lower plenum of the Reactor Structure Assembly (RSA) and bottom of the GCCT. Thus, cooling water passing the core can flow to the GCCT through the RHRP to remove core decay heat.

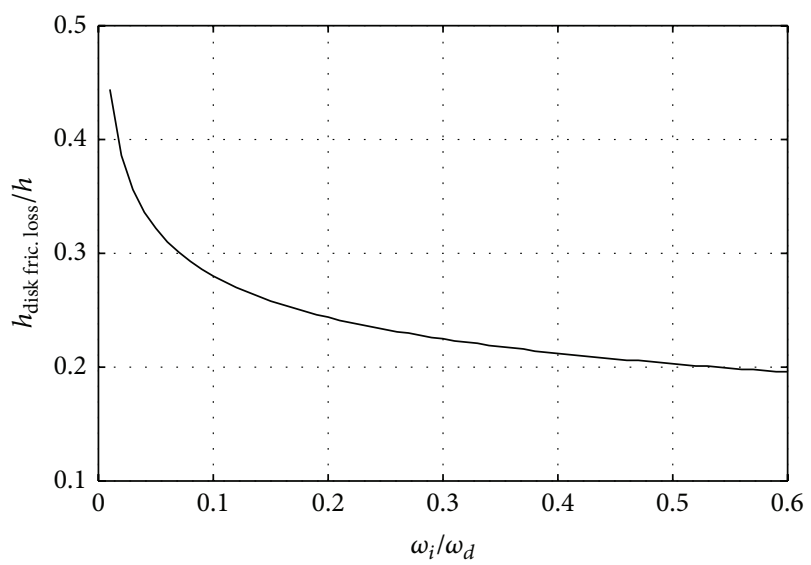

FIgURE 3: Proportion of the disk friction loss to the pump head raise with the impeller rotating speed.

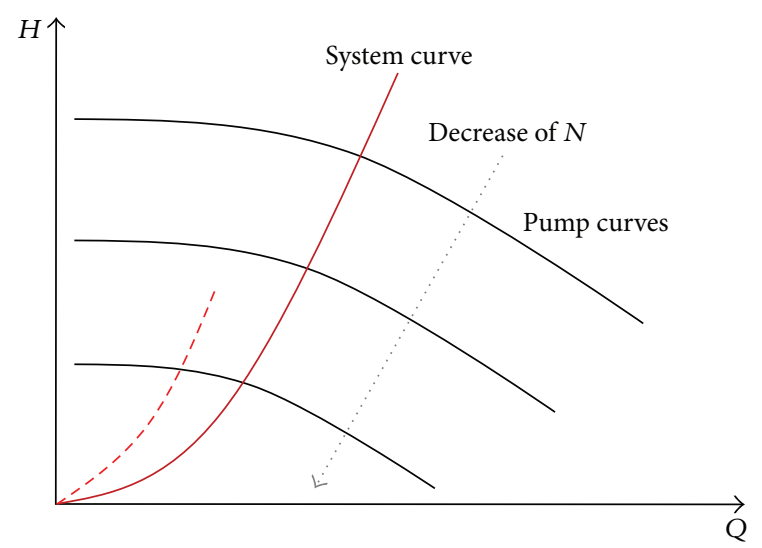

FIGURE 4: Change of the system resistance curve with the decrease of the flow rate.

Additionally, a Pool Water Cooling and Purification System (PWCPS) was designed to improve the safety and usability of the research reactor, as shown in Figure 5. A PWCPS consists of a pump, a flow control valve, a heat exchanger, a filter, an ion exchanger, and related pipes. A Decay Wall (DW) is installed to separate a discharge area from the RHRP and a suction area of the PWCPS pump because the cooling water passing the core has a very high radiation level. Namely, cooling water discharged from the RHRP stays for a sufficiently long time inside the GCCT before entering the PWCPS to decay out N-16, which has a high radiation level but short half-life. As a result, the cooling water inside the PWCPS has a low radiation level, and the design of the PWCPS is easy and cheap.

Here, the reactor pool, GCCT, and RHRP shall be designed as the safety class, but the PWCPS will be a nonsafety class because it is not directly related to reactor safety.

2.3.1. PCS Operation Stage. Figure 5 shows the reactor off, PCP off, and PWCPS pump off states of an open-pool type research reactor. The reactor pool and GCCT have the same 


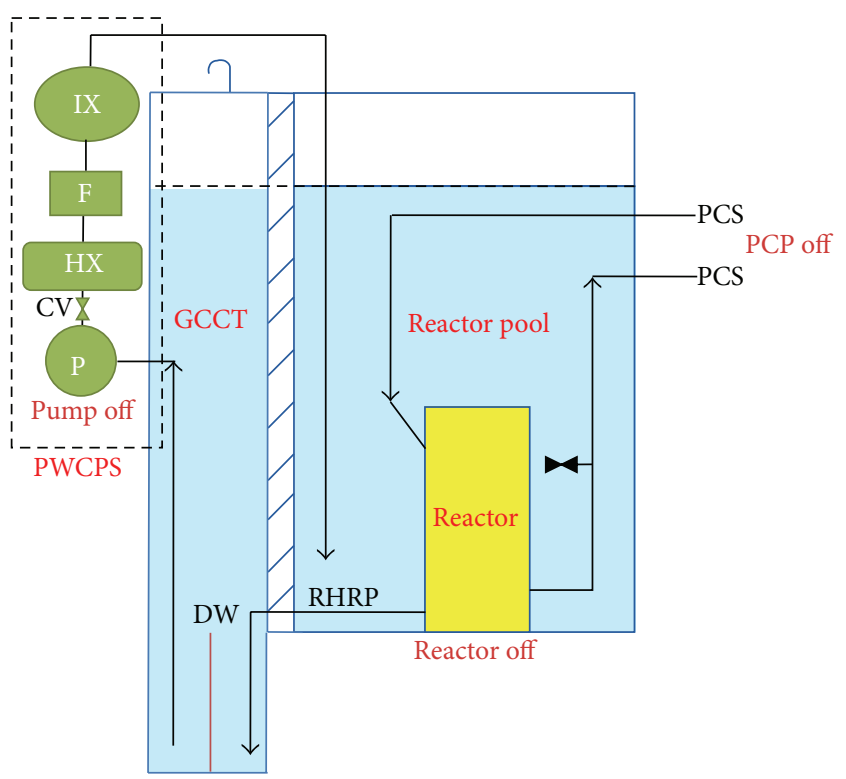

GCCT: gravity core cooling tank

RHRP: residual heat removal pipe

DW: decay wall

PWCPS: pool water cooling and purification system

P: pump

CV: control valve

HX: heat exchanger

F: filter

IX: ion exchanger

FIGURE 5: Schematic diagram of PRHRS using GCCT.

pool water levels because cooling water can move freely through the RHRP between the two pools.

After the PCP is turned on, the water level of the GCCT gradually decreases because some water moves from the GCCT to the reactor pool through the RHRP. An increase of the reactor pool water level is small because the surface area of the reactor pool is much larger than that of GCCT. Finally, the two pools have a water level difference, as shown in Figure 6, and the flow of cooling water through the RHRP is stopped. Here, the hydrostatic head by the water level difference is the same as the pressure drop through the core.

2.3.2. Normal Power Operation Stage. PWCPS is turned on after the PCS, reactor pool, and GCCT are in stable operation conditions. Cooling water flows in the PWCPS from the GCCT and is discharged into the reactor pool after passing the pump, heat exchanger, filter, and ion exchanger of the PWCPS. Some reactor pool water is suctioned into the reactor through the top opening of the RSA, mixed with PCS water, and passed the core. Some of the heated cooling water at the lower plenum of the RSA moves into the GCCT through RHRP. After PWCPS is stable with the balanced mass flow rates, the reactor will start a power operation. A flow diagram for normal power operation conditions is shown in Figure 7.

When PCS water is discharged into the RSA, some PCS water will be released into the reactor pool through the top

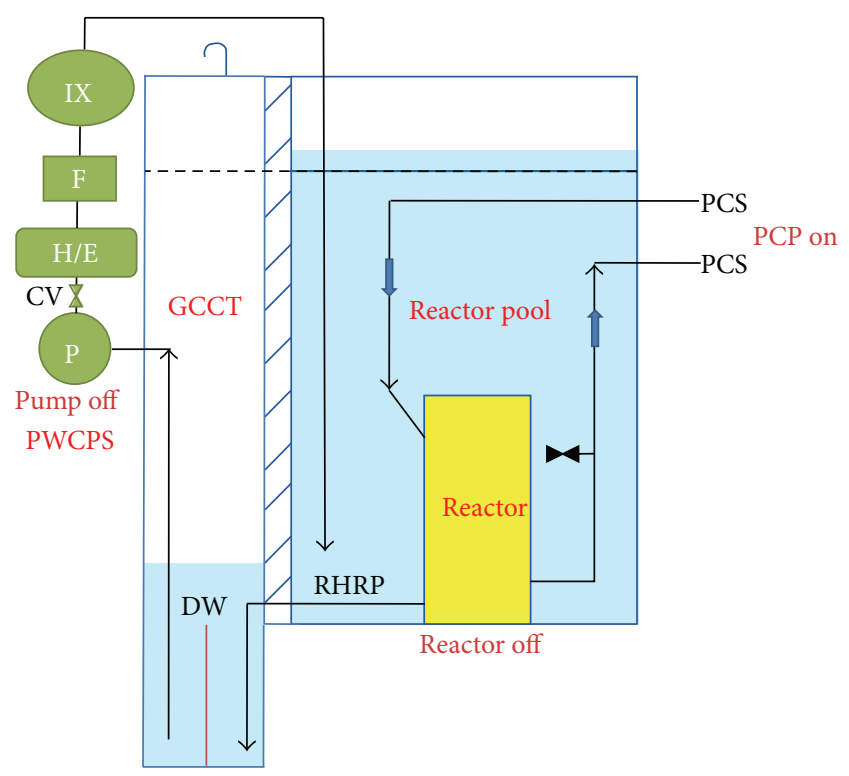

FIGURE 6: PCS operation.

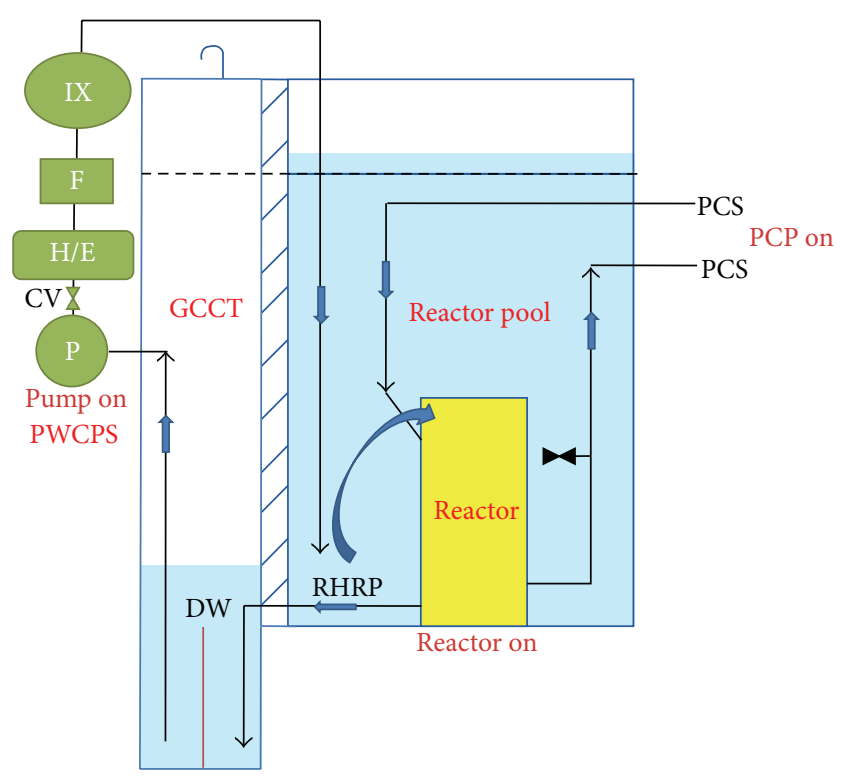

FIGURE 7: Reactor operation.

opening of the RSA owing to collisions between the flows and structures. It will generate some flow in the upper section of the reactor pool above the reactor, and this flow will make the radiation material move on the surface of the reactor pool. In this case, the reactor operation will be impossible because of a high pool top surface radiation limit. However, the suction flow of the reactor pool water through the top opening of the RSA blocks the released PCS flow, and the pool top surface radiation level is low. In addition, an extra purification system for a PCS is not required because the purified water from a PWCPS is mixed with PCS water.

2.3.3. Normal Shutdown Stage. Figure 8 shows the pool water level and flow when nuclear fuel or targets for isotope 


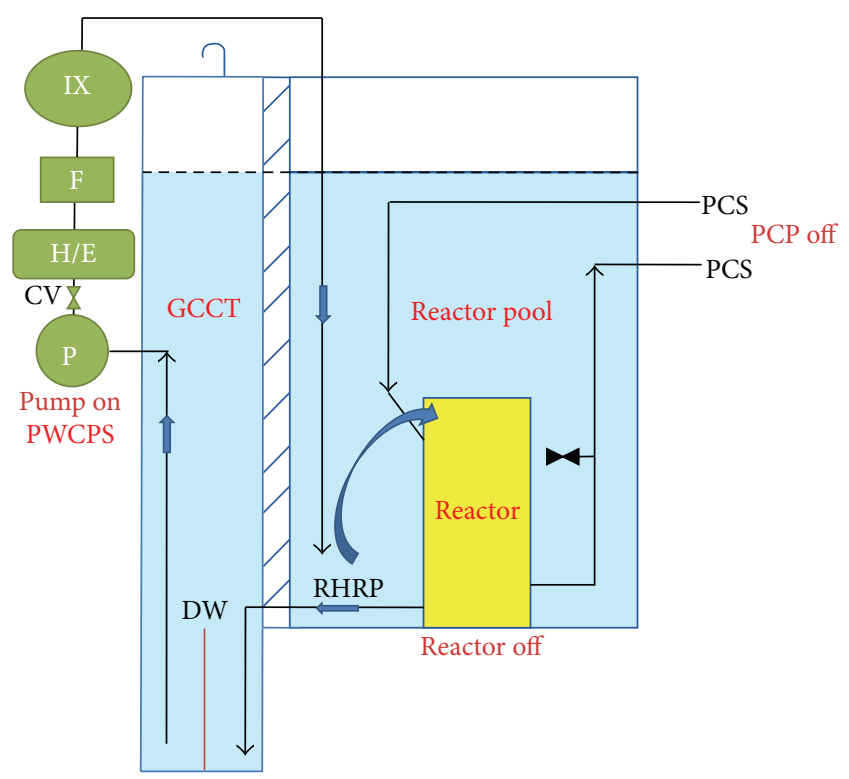

FIGURE 8: Reactor normal shutdown.

production are changed after a reactor normal shutdown. The PCP is turned off after the reactor is shut down, and the core differential pressure is decreased. Then, some reactor pool water moves through the core and RHRP, and the core decay heat is removed. Finally, the reactor pool and GCCT have the same water levels. Because PWCPS is still in operation, the core downward flow is continually maintained. It can remove the core decay heat and heat waves actively. Here, a check valve shall be installed in the PCS pipe to protect the backflow and confirm that the RHRP flow is identical with the core downward flow. In addition, a flow control valve is designed at the downstream of the PWCPS pump owing to water level changes in GCCT.

2.3.4. Reactor Accident Condition. Under reactor accident conditions, the reactor is shut down, and the PCP is quickly turned off. Even though PWCPS can remove the core decay heat, this system shall be neglected for the safety analysis because it is a nonsafety class. The water level difference between the reactor pool and GCCT maintains the core downward flow naturally, as shown in Figure 9, and the flow stops when two pool levels are equal. Then, the flap valves on the PCS pipe inside the reactor pool are automatically opened by gravity force. The decay heat is removed by natural circulation through the flap valves, lower plenum, and the core to the pool. It is important to determine the sizes of GCCT and RHRP considering the decay heat curve and natural circulation performance.

\section{Preliminary Design of Passive Residual Heat Removal System}

An open-pool research reactor was preliminarily designed in this paper. It has $15 \mathrm{MW}$ of thermal power, a $30 \mathrm{~m}^{2}$ reactor pool area, a $40 \mathrm{~m}^{2}$ service pool area, and $11 \mathrm{~m}$ water levels under normal power operation conditions. Normally,
TABle 1: ANSI/ANS-5.1-2005, Decay Heat Power in Light Water Reactors.

\begin{tabular}{lc}
\hline Time after shutdown(s) & $P / P_{0}$ \\
\hline $1.00 E+00$ & $6.065 E-02$ \\
$1.00 E+01$ & $4.730 E-02$ \\
$1.00 E+02$ & $3.193 E-02$ \\
$1.00 E+03$ & $1.980 E-02$ \\
$1.00 E+04$ & $9.721 E-03$ \\
\hline
\end{tabular}

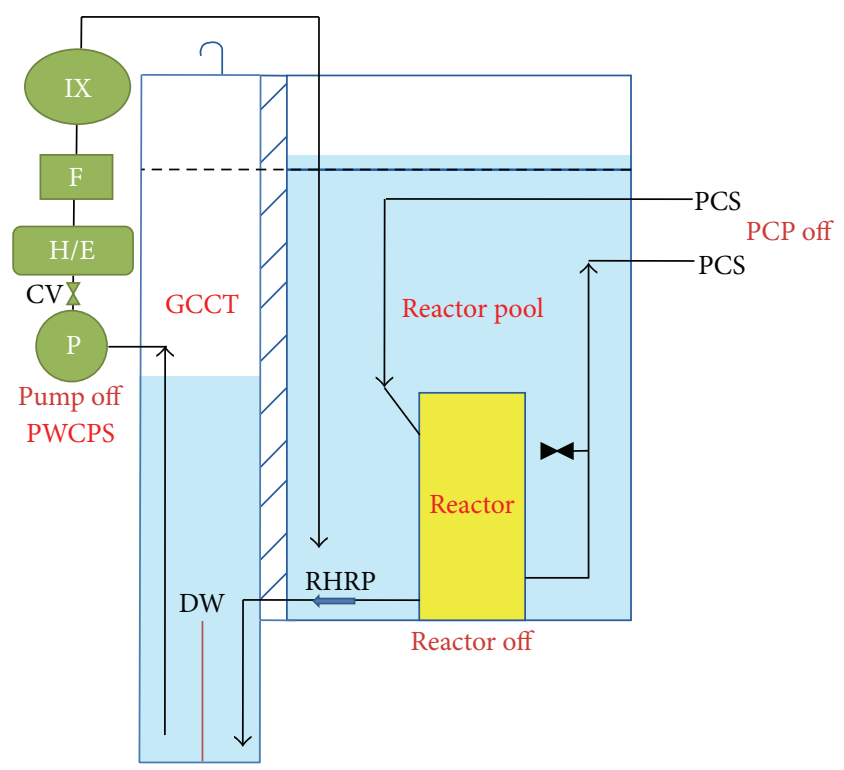

Figure 9: Reactor accident.

the pool gate between the reactor pool and service pool was opened. The flow rate of the PCS was $600 \mathrm{~kg} / \mathrm{s}$, and the pressure drop through the core was $110 \mathrm{kPa}$. During normal reactor operation, the rector pool and service pool therefore had an $11 \mathrm{~m}$ water level, and the GCCT had a $0 \mathrm{~m}$ water level. Here, the inlet/outlet coolant temperature difference at the core was $6^{\circ} \mathrm{C}$ from the heat balance. Consider

$$
Q=\dot{m} C_{p} \Delta T .
$$

To obtain a decay of the core, ANSI/ANS-5.1-2005 was considered as shown in Table 1 [11]. At 1 second after the reactor shutdown, $6 \%$ of full power was considered as a decay heat of the core and was $900 \mathrm{~kW}$. To consider the hot channel effect, we assumed that the required heat removal capacity was conservatively double, that is, $1800 \mathrm{~kW}$ at 1 second. The decay heat was decreased to $3.2 \%$ and $2 \%$ of full power at 100 and 1000 seconds after a shutdown, and the required heat removal capacities were 960 and $600 \mathrm{~kW}$ considering the hot channel effect, respectively. In addition, if the inlet/outlet coolant temperature difference at the core was assumed to be $6^{\circ} \mathrm{C}$, the required flow rates were $71 \mathrm{~kg} / \mathrm{s}, 38 \mathrm{~kg} / \mathrm{s}$, and $22 \mathrm{~kg} / \mathrm{s}$, respectively.

At first, a flywheel with $160 \mathrm{~kg} \cdot \mathrm{m}^{2}$ inertia was considered in the PCP for the initial cooling of the core after a reactor shutdown. A coastdown flow by the flywheel is shown in 


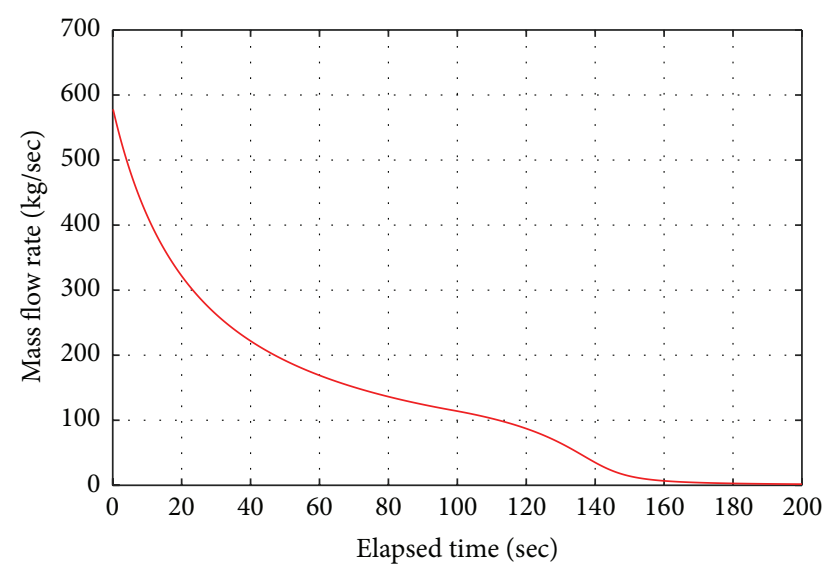

FIgURE 10: Coastdown of the pump with time (designed).

Figure 10. It was sufficient to maintain the downward flow to remove the core decay heat until approximately 130 seconds.

Next, a GCCT with a $5 \mathrm{~m}^{2}$ pool area and a 5-inch RHRP was considered. The friction coefficient from the reactor pool to the GCCT was 8 considering the pressure drop through the core and RHRP. The velocity at the RHRP was calculated from the Bernoulli equation:

$$
v=\sqrt{\frac{2 g\left(H_{1}-H_{2}\right)}{1+K_{12}}} .
$$

The flow rate through the RHRP was calculated as follows:

$$
\dot{m}=\rho A_{\mathrm{RHRP}} v .
$$

The reactor pool water level gradually decreased and the GCCT water level increased. The flow disappeared when the two water levels were the same. To consider these level changes, the following simple equations are used:

$$
\begin{aligned}
& H_{1}=H_{\mathrm{Rx}}-\int \frac{\dot{m}(t)}{\rho A_{\mathrm{Rx}+\mathrm{Ser}}} d t, \\
& H_{2}=H_{\mathrm{GCCT}}+\int \frac{\dot{m}(t)}{\rho A_{\mathrm{GCCT}}} d t .
\end{aligned}
$$

Figures 11 and 12 show the flow rate from the reactor pool to the GCCT along with their water levels. The flow rate was decreased linearly but was higher than the required flow rates until 1000 seconds. The flow stopped at 1624 seconds, and the final water levels were identically $10.3 \mathrm{~m}$. Here, we should regard the flow through the RHRP to start from a zero flow rate. Therefore, the PCS flow inertia and flywheel should be used to cool the core immediately after a reactor shutdown. The GCCT may be capable of applying an intermediate cooling method.

Regarding the characteristics of subgroup (A) described in Section 2.1, we can think that a natural circulation flow can be removed at $300 \mathrm{~kW}$ or less of decay heat after 1000 second, and the flow reversal from downward to upward at the core may be permitted. For long-term cooling, the flap valve will be opened at around 1000 seconds after a shutdown to generate natural circulation. Actually, a study on natural circulation is another research topic.

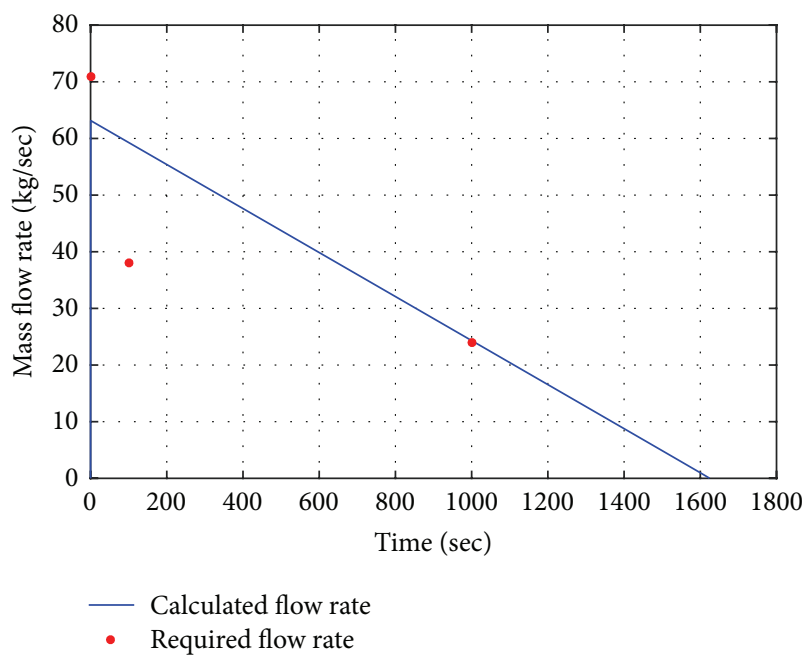

FIgURE 11: Flow rate through the RHRP.

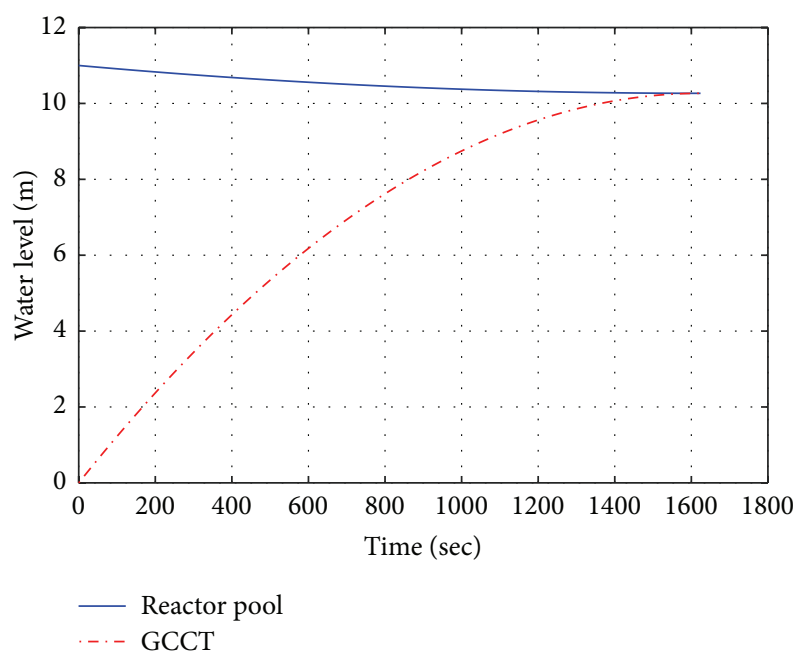

FIGURE 12: Water levels of reactor pool and GCCT.

\section{Discussions}

An innovative PRHRS for an open-pool type research reactor is suggested, and the pump flywheel and GCCT are studied to design this system for a research reactor with $15 \mathrm{MW}$ of thermal power. The pump coastdown mechanism is described by using the affinity laws of the pump and the angular momentum of the flywheel. In addition, the modified hydraulic efficiency model is suggested to predict the continuous time of the coastdown. A GCCT beside the reactor pool with a RHRP connecting two pools was developed and designed preliminarily as a PRHRS for an open-pool type research reactor. The GCCT can remove the decay heat of the core with the flywheel of the PCP and the flap valves in an accident situation of the reactor. It is very simple to design and cheap to construct. Additionally, a nonsafety but active residual heat removal system, named PWCPS, is applied with the GCCT. It can improve the usability of the research reactor by removing the thermal 
waves and purify the reactor pool, PCS, and GCCT. Moreover, it can reduce the pool top radiation level.

\section{Nomenclature}
A: Area, $\mathrm{m}^{2}$
$D$ : Impeller diameter, $\mathrm{m}$
$g$ : Gravity acceleration, $\mathrm{m} / \mathrm{s}^{2}$
$h$ : Total head raise, $m$
$H$ : Head or height, $\mathrm{m}$
I: Moment of inertia, $\mathrm{kg} \cdot \mathrm{m}^{2}$
$K$ : Friction coefficient
$\dot{m}$ : Mass flow rate, $\mathrm{kg} / \mathrm{s}$
$N$ : Rotational speed, rpm
$P$ : Pump power, $\mathrm{W}$
Q: Volumetric flow rate, $\mathrm{m}^{3} / \mathrm{s}$
T: Temperature, ${ }^{\circ} \mathrm{C}$
$t$ : Time, $\mathrm{s}$
$v$ : Velocity, $\mathrm{m} / \mathrm{s}$.

\section{Greeks}

$\alpha$ : Angular acceleration, $\operatorname{rad}^{2} / \mathrm{s}$

$\eta$ : Efficiency

$\rho$ : Density, $\mathrm{kg} / \mathrm{m}^{3}$

$\omega:$ Angular velocity, $\mathrm{rad} / \mathrm{s}$

$\Delta$ : Difference.

\section{Subscripts}

$d: \quad$ Design point

GCCT: Gravity core cooling tank

$h$ : Hydraulic

$i$ : $\quad$ Iteration number

RHRP: Residual heat removal pipe

Rx: Reactor pool

Ser: $\quad$ Service pool.

\section{Conflict of Interests}

The authors declare that there is no conflict of interests regarding the publication of this paper.

\section{Acknowledgment}

This work was supported by the National Research Foundation of Korea (NRF) grant funded by the Korean Government (MSIP) (no. NRF-2012M2C1A1026909).

\section{References}

[1] NRC, "Backgrounder on Research and Test Reactors," http:// www.nrc.gov/reading-rm/doc-collections/fact-sheets/research -reactors-bg.html.

[2] W. J. Brynda, P. R. Lobner, R. W. Powell, and E. A. Straker, Design Guide for Category III Reactors: Pool Type Reactors, Brookhaven National Laboratory, Upton, NY, USA, 1978.

[3] H. G. Yoon, Y. C. Park, K. W. Seo, D. Y. Chi, and I. S. Yoo, "Prediction model of the HANARO primary cooling pump coastdown," in Proceedings of the Korean Nuclear Society Spring Meeting, Taebaek, Republic of Korea, October 2011.

[4] S. L. Dixon, Fluid Mechanics Thermodynamics of Turbomachinery, Elsevier, New York, NY, USA, 2013.

[5] S. Lazarkiewicz, Impeller Pumps, Pergamon Press, New York, NY, USA, 1965.

[6] H. W. Oh and M. K. Chung, "Optimum values of design variables versus specific speed for centrifugal pumps," Proceedings of the Institution of Mechanical Engineers, Part A: Journal of Power and Energy, vol. 213, no. 3, pp. 219-226, 1999.

[7] J. W. Daily and R. E. Nece, "Chamber dimension effects on induced flow and frictional resistance of enclosed rotating disks," Journal of Basic Engineering, vol. 82, no. 1, pp. 217-232, 1960.

[8] B. R. Munson, D. F. Young, and T. H. Okiishi, Fundamentals of Fluid Mechanics, John Wiley \& Sons, 4th edition, 2002.

[9] K. Y. Lee, D. Y. Chi, S. H. Kim, K. W. Seo, and J. Yoon, "Passive residual heat removal system of an open-pool type research reactor using gravity core cooling tank," in Proceedings of the European Research Reactor Conference (RRFM '14), pp. 593-599, Ljubljana, Slovenia, March-April 2014.

[10] K. Y. Lee, S. H. Kim, K. W. Seo, D. Y. Chi, and J. Yoon, "Residual Heat Removal System for Research Reactor," Article ID 1457293, Korea patent 10-1457293, 2014.

[11] ANSI/ANS-5.1-2005: Decay Heat Power in Light Water Reactors (with erratum). 


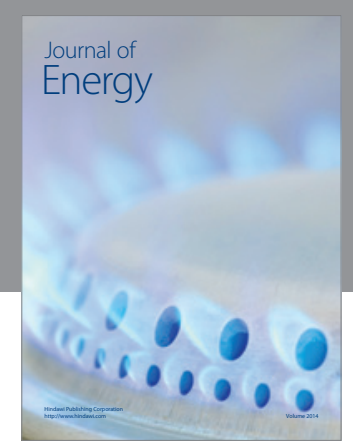

Journal of

Industrial Engineering
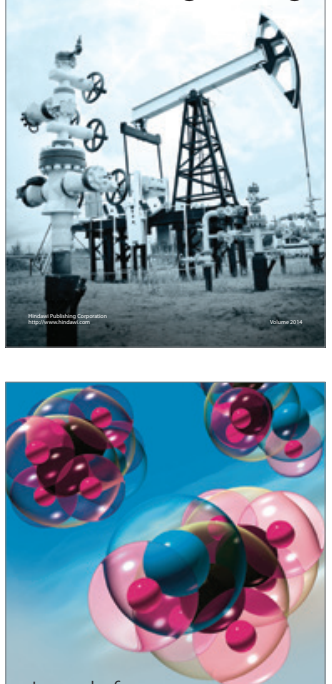

Fuels
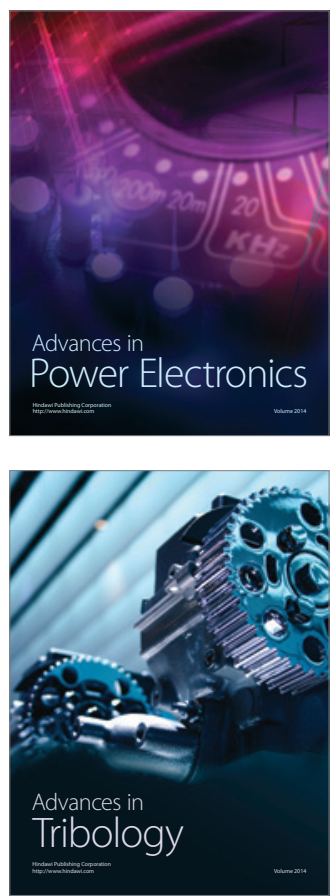

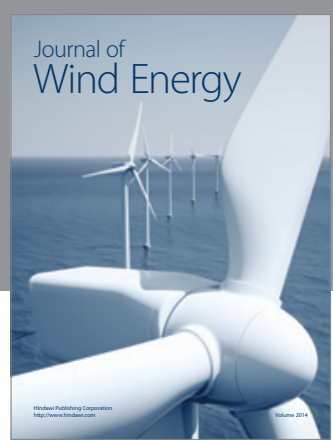

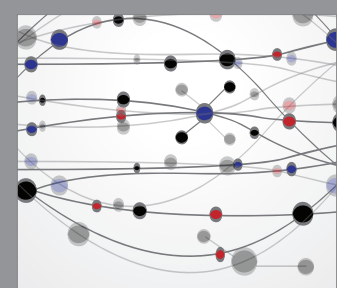

The Scientific World Journal

Submit your manuscripts at http://www.hindawi.com

Journal of

Structures
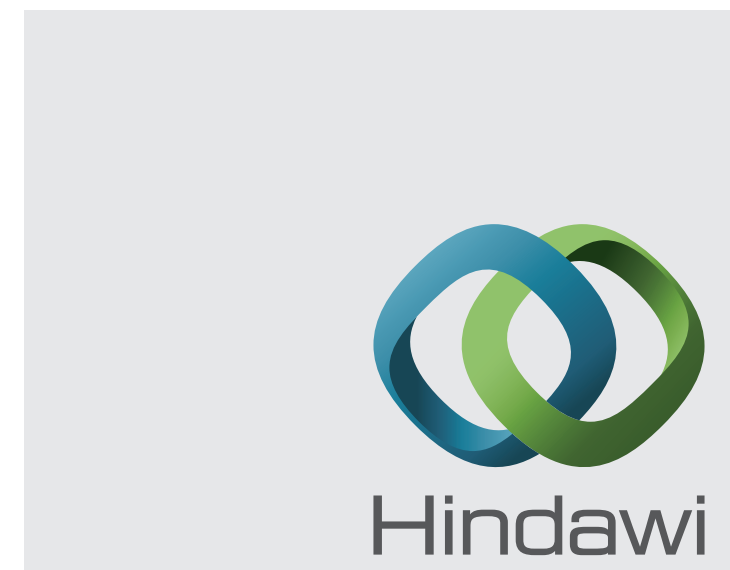

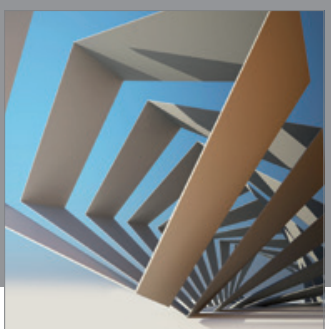

Rotating

Machinery
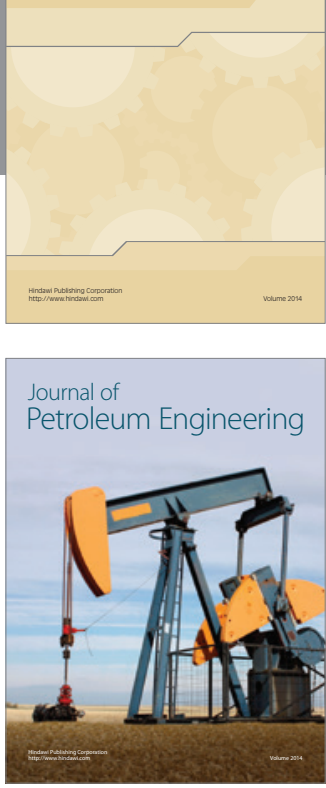

Journal of

Solar Energy
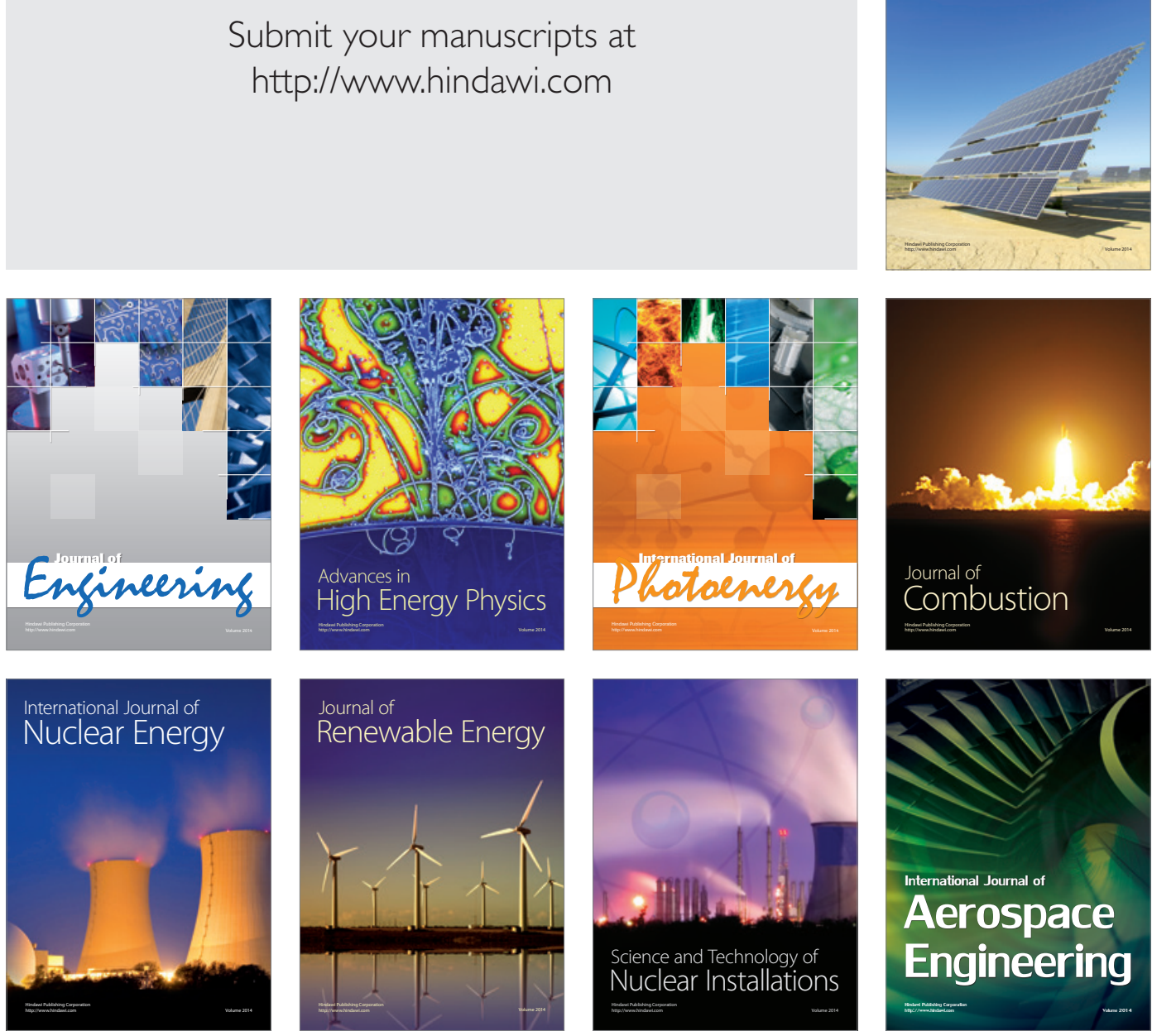